\section{Phototropism in Porcellana Larvæ}

VARIOUs explanations of the interesting phenomena classed as 'tropisms' have, from time to time, been put forward; ranging from Loeb's completely mechanical hypothesis ${ }^{1}$ to the point of view of Russell $^{2}$, who maintains that what we see is the attempt of the animal to escape from an unusual situation; he calls this a 'flight response'.

Neither of these points of view is entirely satisfactory. It is not clear why the nature of a 'flight response' should vary with, say, the temperature, as it must in those cases where the sign of phototropism is changed by a slight rise in temperature; and, again, 'flight response' does not appear to fit in with Clarke's important work on Daphnia ${ }^{3}$.

Loeb's theory of response to light which he called the "Muscle Tension Theory of Heliotropism" (loc. cit., p. 54) has had to be modified in the light of work $^{3}$ which has shown that orientation is based on a mechanism entirely distinct from that of certain other aspects of phototropism. As a rule it is difficult to distinguish between (1) movement and (2) orientation, in respect to light ; but the larvæ of Porcellana give a good example in which these processes are distinet.

The reactions of Porcellana larvæ to light have been described by Spooner ${ }^{4}$. He conoluded that the behaviour of these larvæ was to be classed as 'phototopotaxis', by which term he indicates a "special type of phototaxis in which the direction of movement is controlled by the direction of the light rays".

Spooner also observed that these Porcellana larvæ sometimes move backwards, instead of forwards, to the source of light. It has been shown elsewhere that if a Porcellana larva is swimming normally towards the source of light and then the direction of light is suddenly reversed, the larva, instead of re-orientating itself to the new source of light, reverses its locomotory mechanism. This failure to re-orientate is correlated with the possession of the very long spines characteristic of these larvæ; for when these spines are cut off, the larvæ, on reversal of the direction of the stimulus, re-orientate immediately.

On Loeb's hypothesis the movements which resulted from stimulation by light were 'forced movements', the animal having no choice over its reactions : the predictable result being brought about mechanically. In the sense that Porcellana larvæ swim towards the light, the movements seen are 'forced'; but the evidence also shows that these movements are not mechanically produced as Loeb's theory holds, but that there is some nervous integration going on which determines the manner in which the response is to be produced.

Finally, it may be pointed out that to deny the presence of a psychological factor does not materially assist in a better understanding of this interesting form of behaviour.

\section{Department of Zoology, \\ University of Glasgow. June 9.}

\section{G. E. H. Foxon.}

1 Loeb, J., "Forced Movements, Tropisms, and Animal Conduct". Philadelphia and London. 1918.

"Russell, E. S., "The Behaviour of Animals". Iondon. 1934.

- Clarke, G. L., J. Exp. Biol., 9, 180-211; 1932.

- Spooner, G. M., J. Mar. Biol. A8s., N.S., 19, No. 1, 385-438; 1933.

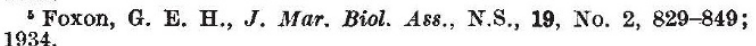

Alleged Influence of Heavy Water on Mould Growth

IAARSON and Barnes ${ }^{1}$ suggest that water containing 0.5 per cent of diplogen exerts a stimulating influence on the growth of moulds. The conclusion is based on the results of experiments carried out partly by themselves and partly by S. L. Meyer ${ }^{2}$ with Aspergillus. Work performed at this Institute casts grave doubt on the validity of their conclusions.

Eight samples of water (diplogen ratio varying from $1: 8$ to $1: 700$ ) prepared by a method involving contact with paraffin, and ten samples of water (diplogen ratio from the highest concentrations down to $1: 800$ ) prepared in the complete absence of organic matter, were repeatedly distilled from alkaline permanganate, and were then exposed to diffuse sunlight, in loosely corked test-tubes. All those samples which had been in contact with paraffin showed the growth of moulds; while those samples prepared in the absence of organic matter remained -and in fact still remain-perfectly clear. A sample of water supplied to us direct from the Ohio Chemical and Manufacturing Company was found to possess a faint but unmistakable odour, which could not be removed by distillation from alkaline permanganate, and which suggested the presence of a trace of organic matter, probably of paraffin nature.

It is clear that the water used in the experiments of Larson and Barnes and of Meyer was twicedistilled 'Ohio'-water. We detected moulds in 'Ohio'-water which we 'purified' in the same way; but samples of water of the same diplogen content which we prepared from electrolyte water supplied by Norsk Hydro Elektrisk Kvaelstofaktieselskab remained quite clear.

It is well known that many organic substances, including paraffins, provide favourable nourishment for the growth of moulds. In the light of the observations recorded here, it would appear more reasonable to attribute this growth to the presence of organic impurity rather than to any 'stimulating' effect of the heavy isotope.

The investigations are being continued.

Institut für physikalische Chemie, R. KLAR. Universität, Frankfurt a. M. June 18.

2 NATURE, 188, 873, June 9, 1934.

Science, 79, 210; 1934.

\section{A Modification of the Gas Circulating Pump}

OcCasronality, while working with the BlackmanBolas pump as modified by Leach ${ }^{1}$, the apparatus ceases to work owing to the valve on the float side not functioning properly. All pumps, whether prepared in the laboratory or obtained from the manufacturers, behaved similarly, and their action was neither so uniform as one would desire it to be, nor could the rate of flow of air be regulated within sufficiently wide limits.

A circulating pump free from these defects is, therefore, desired. A special type of mercury valve has been devised to replace the float and valve arrangements of the Blackman-Bolas pump, and the pump so modified has been found quite satisfactory. The complete pump with the mercury valve is shown in the accompanying diagram (lig. 1).

Enough mercury should be put in to reach a little above the tip of the inner narrow tube. This gives 